\title{
Overexpression of lipoprotein lipase in transgenic rabbits leads to increased small dense LDL in plasma and promotes atherosclerosis
}

Tomonaga Ichikawa ${ }^{1}$, Shuji Kitajima ${ }^{2}$, Jingyan Liang ${ }^{1}$, Tomonari Koike ${ }^{1}$, Xiaofei Wang ${ }^{1}$, Huijun Sun ${ }^{1,3}$, Mitsuyo Okazaki ${ }^{4}$, Masatoshi Morimoto ${ }^{2}$, Hisataka Shikama ${ }^{5}$, Teruo Watanabe ${ }^{6}$, Nobuhiro Yamada ${ }^{7}$ and Jianglin Fan ${ }^{1}$

${ }^{1}$ Cardiovascular Disease Laboratory, Department of Pathology, Institute of Basic Medical Sciences, University of Tsukuba, Tsukuba, Japan; ${ }^{2}$ Analytical Research Center for Experimental Sciences, Saga University, Saga, Japan; ${ }^{3}$ Department of Pharmacology, Dalian Medical University, Dalian, China; ${ }^{4}$ Laboratory of Chemistry, College of Liberal Arts and Sciences, Tokyo Medical and Dental University, Tokyo, Japan; ${ }^{5}$ Yamanouchi Pharmaceutical Company, Tsukuba Research Institute, Tsukuba, Japan; ${ }^{6}$ Saga University, Saga, Japan and ${ }^{7}$ Division of Metabolism and Endocrinology, Institute of Clinical Medicine, University of Tsukuba, Tsukuba, Japan

Lipoprotein lipase (LPL) is a key enzyme in the hydrolysis of triglyceride-rich lipoproteins. Previous studies using transgenic mice and rabbits have demonstrated that high level of LPL activity in adipose and skeletal muscle protects against diet-induced hypercholesterolemia and subsequently prevents aortic atherosclerosis. However, it is unknown, per se, whether increased LPL activity itself is antiatherogenic, or whether the antiatherogenic effect of LPL is dependent upon the LPL lipid-lowering effect. To address this issue, we fed LPL transgenic and littermate rabbits diets containing different amounts of cholesterol (0.3-0.6\%) adjusted to maintain their plasma cholesterol concentrations at similarly high levels for 16 weeks. We analyzed their lipoprotein profiles and compared their susceptibility to atherosclerosis. The results showed that the overexpression of LPL in transgenic rabbits reduced remnant lipoproteins ( $\beta$-VLDL, $d<1.006 \mathrm{~g} / \mathrm{ml})$ but concomitantly led to a significant increase of the large $(d=1.02-1.04 \mathrm{~g} / \mathrm{ml})$ and small LDLs $(d=1.04-1.06 \mathrm{~g} / \mathrm{ml})$ compared to the amounts in control rabbits. Furthermore, we found that with equally high hypercholesterolemia, transgenic rabbits developed 1.8-fold more extensive aortic atherosclerosis than control rabbits. To examine the hypothesis that altered lipoprotein profiles may be responsible for the enhanced atherosclerosis in transgenic rabbits, we studied the atherogenic properties of apoB-containing lipoproteins in vitro. These studies revealed that small-sized LDLs of transgenic rabbits were more susceptible to copper-induced oxidation and had higher affinity to biglycan than large remnant lipoproteins. We conclude, therefore, that LPL exerts a dual function in terms of its atherogenicity, namely antiatherogenicity, through enhancing receptormediated remnant lipoprotein catabolism and proatherogenicity via the generation of a large amount of smallsized LDLs. At an equal atherogenic-cholesterol level, small and dense LDLs are more atherogenic than large remnant lipoproteins.

Laboratory Investigation (2004) 84, 715-726, advance online publication, 3 May 2004; doi:10.1038/labinvest.3700102

Keywords: atherosclerosis; hypercholesterolemia; lipoprotein lipase; macrophage; remnant lipoprotein; small dense LDL; transgenic rabbit

Correspondence: Dr J Fan, Department of Pathology, Institute of Basic Medical Sciences, University of Tsukuba, Tsukuba 3058575, Japan.

E-mail: J-LFAN@md.tsukuba.ac.jp

Received 26 December 2003; revised 18 February 2004; accepted 19 February 2004; published online 3 May 2004
Lipoprotein lipase (LPL) plays a crucial role in lipid metabolism and transport by catalyzing the hydrolysis of triglyceride-rich (TG-rich) lipoproteins such as chylomicrons and very-low-density lipoproteins (VLDLs) and causes a wide variety of alterations in lipoprotein metabolism. Through the hydrolysis of TG in these particles, LPL converts these TG-rich lipoproteins to cholesterol-rich small and dense 
lipoproteins including chylomicron remnants, intermediate-density lipoproteins (IDLs) and low-density lipoproteins (LDLs). This process also results in the generation of surface remnants, which give rise to high-density lipoproteins (HDLs). In addition, LPL is essential for the clearance of apoB-containing lipoproteins and increased LPL activity may lead to the enhancement of the hepatic removal of the lipolyzed lipoproteins. ${ }^{1-3}$

The effects of LPL on atherosclerosis have been controversial. In general, LPL via its action on circulating fasting and postprandial lipoproteins is viewed as an antiatherogenic enzyme. This notion has been supported by the atheroprotective actions of a compound (NO-1886) that raises LPL activity in cholesterol-fed animals. ${ }^{4,5}$ In addition, systemically elevated expression of LPL in either LDL receptor or apoE knockout (KO) mice was associated with protection against atherosclerosis. ${ }^{6,7}$ Furthermore, patients who are deficient in LPL or have LPL mutations develop premature atherosclerosis, which favors a hypothesis that LPL may be an antiatherogenic enzyme. ${ }^{8,9}$ On the other hand, LPL is also expressed by macrophages and smooth muscle cells in atherosclerotic lesions, ${ }^{10,11}$ suggesting that LPL may be potentially proatherogenic. The first theory implicating LPL in atherosclerosis was proposed by Zilversmit ${ }^{12} 30$ years ago. He postulated that high local concentrations of cholesterol-rich remnants and LDLs, produced by the action of LPL on VLDL and chylomicrons at the vascular endothelium, would be taken up into the arterial wall and thus lead to the deposition of cholesterol. ${ }^{12}$ However, this hypothesis has not been fully verified experimentally. Until now, most studies have focused on whether LPL affects cholesterol-rich remnant clearance in an antiatherogenic manner ${ }^{6}$ or whether macrophage-derived LPL in the arterial wall is proatherogenic. $^{13,14}$ The antiatherogenic roles of LPL are mainly mediated by the enhanced hepatic clearance of remnant lipoproteins ${ }^{15}$ which leads to lowering plasma cholesterol, ${ }^{16}$ whereas the proatherogenic functions of LPL are mainly attributed to the noncatalytic function of vascular cellderived LPL, which may not only promote the retention of atherogenic lipoproteins in the inti$\mathrm{ma}^{17,18}$ but also aid in the uptake of lipoproteins by macrophages as well. ${ }^{19}$ Moreover, the process of the hydrolysis of TG-rich lipoproteins by LPL also leads to the generation of IDLs and the enhancement of the conversion of large LDLs to small and dense LDLs. ${ }^{3}$ It has not been defined whether small-sized LDLs generated by LPL are atherogenic. In fact, accumulating evidence from epidemiological and clinical studies has shown that a preponderance of small dense LDLs is linked to increased risk of myocardial infarction and the presence of coronary artery disease. ${ }^{20,21}$ Studies using genetically modified animals have revealed that remnant lipoproteins and LDLs are actually different in terms of atherogenicity. ${ }^{22,23}$ Although LPL is protective against cholesterol-diet-induced atherosclerosis in LDL receptor and apoE KO mice, ${ }^{6,7}$ studies of these mice did not demonstrate a significant increase of small LDLs in LPL transgenic animals and thus failed to provide a clear answer about whether LPL-generated small LDLs are atherogenic.

Our laboratory has been using transgenic rabbits to study atherosclerosis. ${ }^{24}$ As an experimental model for the study of lipid metabolism and atherosclerosis, rabbits have several advantages over mice. For example, rabbits (LDL-mammals) have higher levels of apoB-containing lipoproteins than mice (HDL-mammals), a lipoprotein profile more like those of humans, and patterns of hepatic apoB100 and intestinal apoB48 synthesis resembling that of humans. Like humans, and unlike mice, rabbits have cholesteryl ester transfer protein, an important enzyme in lipoprotein metabolism and atherosclerosis. ${ }^{25}$ Moreover, cholesterol-fed rabbits develop remnant-rich hypercholesterolemia and atherosclerosis. Recently, we reported that systemically increased LPL expression in transgenic rabbits protected against cholesterol-diet-induced hypercholesterolemia and led to the subsequent inhibition of aortic atherosclerosis. ${ }^{16}$ However, in that study, plasma cholesterol levels $(<250 \mathrm{mg} / \mathrm{dl})$ in transgenic rabbits were significantly lower than those in control rabbits, and therefore it is unknown whether increased LPL activity itself would directly affect atherogenicity or whether the antiatherogenicity of increased LPL is completely dependent upon LPL's lipid-lowering effect. In order to address these issues, we performed the following experiments in which transgenic and control rabbits were induced to develop equally high hypercholesterolemia. We characterized the lipoprotein profiles and compared the susceptibility to atherosclerosis in these animals and found that at a given total plasma cholesterol level, overexpression of LPL in transgenic rabbits led to reduced accumulation of remnant lipoproteins but significantly increased small and dense LDL particles associated with greater aortic atherosclerosis. Our experiments provide fresh insights into the intrinsic roles of LPL in lipoprotein modulation and atherosclerosis.

\section{Materials and methods}

\section{Human LPL Transgenic Rabbits}

Transgenic rabbits overexpressing human LPL (hLPL) under the control of $\beta$-actin promoter were generated as reported previously. ${ }^{16}$ The hLPL transgene was largely expressed in multiple tissues such as adipose tissue, heart, and skeletal muscles, as shown by Northern blot analysis (data not shown). In the current study, 12 male transgenic and 16 littermate control rabbits at the age of 4-5 months were used. They were fed a diet containing 0.3$0.6 \%$ cholesterol and $3 \%$ soybean oil. Since transgenic rabbits are less responsive to cholesterol-diet 
feeding and thus have lower levels of plasma cholesterol than do control rabbits on the same cholesterol diet, ${ }^{16}$ we fed transgenic and control rabbits diets containing different amounts of cholesterol in order to generate equally high hypercholesterolemia. To achieve this, we measured plasma lipids weekly and adjusted the cholesterol content of the diet according to the changes of plasma cholesterol of each individual animal. The animals were fed each diet ad libitum. All animal experiments were performed with the approval of the Animal Research Committee of the University of Tsukuba, Japan.

\section{Plasma Lipid and Lipoprotein Analysis}

Blood samples were collected from rabbits in the morning. The plasma total cholesterol (TC) and triglycerides (TG) were analyzed weekly using Wako assay kits (Wako Chemicals, Osaka). The enzymatic activity of LPL in the postheparin plasma was determined using ${ }^{14} \mathrm{C}$-labeled triolein emulsion substrate as described before. ${ }^{16}$ For the determination of the apolipoprotein distribution in transgenic rabbit lipoproteins, plasma lipoproteins were isolated by sequential ultracentrifugation as described. ${ }^{26}$ These lipoprotein fractions were subjected to agarose gel electrophoresis and stained with Fat red $7 \mathrm{~B}$, or transferred to a nitrocellulose membrane for immunoblotting with goat anti-apoB, -apoAI, and -apoE polyclonal Abs (Rockland Inc., Gilbertsville, PA, USA) and immunocomplexed proteins were identified by reaction with a horseradish peroxidase-conjugated horse $\mathrm{Ab}$ to goat IgG, followed by enhanced chemiluminescent detection (ECL kit, Amersham, Arlington Heights, IL, USA). TC and TG contents in each density fraction were measured using Wako assay kits. The distribution of TC and TG within the plasma lipoproteins was also determined by high-performance liquid chromatography (HPLC) on two columns of TSK gel Lipopropak XL (TOSOH, Tokyo) as described before. ${ }^{7,16}$ For the evaluation of the lipoprotein size, isolated lipoprotein density fractions from rabbits fed a cholesterol diet for 8 and 16 weeks were examined by negative-stain electron microscopy as described. ${ }^{16}$

\section{Quantification of Aortic Atherosclerosis}

After 16 weeks of cholesterol diet, the rabbits were killed by an overdose injection of sodium pentobarbital solution. The whole aortas were stained with Sudan IV for evaluation of the gross size of the atherosclerosis. ${ }^{27}$ For microscopic evaluation of the lesion areas, the entire aortic arch (approximately $1 \mathrm{~cm}$ long) was serially cut into sections at $1-\mathrm{mm}$ intervals (10-12 sections/aorta) and embedded in paraffin and stained with hematoxylin-eosin and Elastica van Gieson. The intimal lesions were measured using a computerized image analysis system and expressed as microscopic lesion areas. ${ }^{28}$ To study cellular components, lipoprotein and LPL deposits in the lesions, we performed immunohistochemical staining using the following monoclonal antibodies against macrophages (RAM-11), smooth muscle $\alpha$-actin (HHF35), oxidized LDL (FOH1a/ DLH3), ${ }^{29}$ and LPL (5D2). ${ }^{30}$

\section{Evaluation of apoB-containing Lipoprotein Oxidizability}

The copper-induced oxidation of lipoproteins was performed by the method described by Kleinveld. ${ }^{31}$ Four fractions of apoB-containing lipoproteins with different densities isolated from the cholesterol-fed control and transgenic rabbits as described above were used within a week. They were lipoproteins with $d<1.006 \mathrm{~g} / \mathrm{ml} \quad(\beta$-VLDL), $\quad d=1.006-1.02 \mathrm{~g} / \mathrm{ml}$ (IDL), $d=1.02-1.04 \mathrm{~g} / \mathrm{ml}$ (IDL and large LDL), and $d=1.04-1.06 \mathrm{~g} / \mathrm{ml}$ (small LDL). These lipoproteins were thoroughly dialyzed against PBS buffer and their lipids and proteins were quantitated. Each lipoprotein $(50 \mu \mathrm{g} / \mathrm{ml})$ was dissolved in phosphate buffer (10 mM, pH 7.4) containing $0.16 \mathrm{M} \mathrm{NaCl}$ and $1 \mu \mathrm{M}$ EDTA. Oxidation was initiated by adding a freshly prepared $\mathrm{CuCl}_{2} \cdot 2 \mathrm{H}_{2} \mathrm{O}$ solution as prooxidant (final concentration $5 \mu \mathrm{M}$ ). The kinetics of lipoprotein oxidation were determined by monitoring the change of the conjugate-diene absorbance at $234 \mathrm{~nm}$ at $37^{\circ} \mathrm{C}$ with a Lambda 5 UV-spectrophotometer (Beckman) equipped with a six-position automated sample changer. Absorbance was recorded every $3 \mathrm{~min}$ consecutively for $5 \mathrm{~h}$. The change in absorbance at $234 \mathrm{~nm}$ vs time could be divided into three consecutive phases: lag time, propagation, and decomposition. Each sample was assessed in triplicate.

\section{Binding Assay of apoB-containing Particles to Biglycan}

We evaluated the binding affinity of differently sized apoB-containing lipoproteins to the extracellular matrix using the method described by Skalen. ${ }^{32}$ For this experiment, we used the four different density fractions of lipoproteins described above. Maxisorp immunoplates (Nunc) were coated with biglycan $(10 \mu \mathrm{g} / \mathrm{ml})$ (Sigma-Aldrich, St Louis, MO, USA) dissolved in HEPES-buffered saline (HBS) overnight at room temperature (RT, $22^{\circ} \mathrm{C}$ ). Then, the plates were blocked with HBS containing $1 \%$ bovine serum albumin (BSA) for $1 \mathrm{~h}$ at RT. The aliquots of isolated lipoproteins in HBS with $2 \mathrm{mM} \mathrm{CaCl}_{2}$ and $2 \mathrm{mM} \mathrm{MgCl}{ }_{2}$ were added to the wells and incubated for $1 \mathrm{~h}$ at RT. After washing $(3 \times), 100 \mu \mathrm{l}$ of goat antiapoB Abs $(0.25 \mu \mathrm{g} / \mathrm{ml})$ (Rockland) in HBS with $0.1 \%$ BSA and $0.02 \%$ Tween 20 was added for $1 \mathrm{~h}$ at RT, and then $100 \mu \mathrm{l}$ of peroxidase-conjugated rabbit antigoat IgG $(0.28 \mu \mathrm{g} / \mathrm{ml})$ (Rockland) was added and 
incubated for $30 \mathrm{~min}$ at RT. Finally, $100 \mu \mathrm{l}$ of $2,2^{\prime}-$ azino-bis- (3-ethylbenziazoline-6-sulfonic acid) (Rockland) was added and incubated for another $30 \mathrm{~min}$ at RT. The absorbance of samples was read using a microplate reader (BioRad Laboratories, Hercules, CA, USA) at $405 \mathrm{~nm}$.

\section{Cholesteryl Ester Formation Assay}

To assess the ability to stimulate the uptake of $\beta$ VLDL and $d<1.04-1.06 \mathrm{~g} / \mathrm{ml}$ lipoproteins by macrophages, we measured cholesteryl ester formation from $\left[1-{ }^{14} \mathrm{C}\right]$ oleate in mouse peritoneal macrophages as described..$^{7,33}$ Briefly, thioglycollate-elicited macrophages were obtained from ICR mice and incubated in $1 \mathrm{ml}$ of medium containing $50 \mu \mathrm{g} / \mathrm{ml}$ of lipoproteins and the $\left[1-{ }^{14} \mathrm{C}\right]$ oleate-BSA complex at $37^{\circ} \mathrm{C}$ for $24 \mathrm{~h}$. Cells were washed once with PBS containing $0.3 \%$ BSA and twice with PBS, followed by extraction of cellular lipids with hexane-isopropanol 3:2 (v/v). Thus, extracted cellular lipids were dried under flowing nitrogen. Cholesterol $\left[1-{ }^{14} \mathrm{C}\right]-$ oleate was separated on slica-coated TLC plates (Merk, Darmstadt, Germany) using a solvent system composed of hexane-diethylether-methanol-acetic acid 85:20:1:1 (v/v/v/v), and its position was identified using iodine vapor. The spots corresponding to cholesteryl esters were scraped and their radioactivities were quantified by liquid scintillation. Cellular proteins were dissolved in $0.1 \mathrm{~N} \mathrm{NaOH}$ and used for the determination of the cellular protein level.

\section{Statistical Analysis}

The lesion analyses and binding assay results were compared using the Mann-Whitney's U-test for nonparametric analysis. Student's $t$-test was used to compare the results of other assays. In all cases, statistical significance was set at $P<0.05$.

\section{Results}

\section{Analyses of Plasma Lipids and Lipoproteins}

Transgenic rabbits had 1.6-fold higher LPL enzymatic activity in postheparin plasma than control rabbits after cholesterol diet feeding $(28.2 \pm$ $10.2 \mu \mathrm{mol} \mathrm{FFA} / \mathrm{ml} / \mathrm{h}$ in transgenic $v s 17.4 \pm 4.8 \mu \mathrm{mol}$ $\mathrm{FFA} / \mathrm{ml} / \mathrm{h}$ in control, $P<0.05)$. Transgenic rabbits fed an adjusted-cholesterol diet showed a similar elevation of plasma TC as control rabbits; however, their TG levels were constantly and significantly lower than those of control rabbits during the whole period of the experiment (Figure 1a and b). HPLC analysis showed that cholesterol was broadly distributed in non-HDL lipoproteins in both transgenic and control rabbits, but the TG contents was dramatically reduced in transgenic rabbits compared to control rabbits (data not shown). Immunoblotting analysis of plasma apoB levels revealed that transgenic rabbits had slightly higher apoB-100 levels than control rabbits, whereas apoB-48 levels were not changed (Figure 1c). In order to determine lipoproteins quantitatively, we further isolated lipoproteins from cholesterol-fed rabbits by sequential density gradient ultracentrifugation and resolved them by $1 \%$ agarose gel electrophoresis. By this method, we were able to compare seven different density fractions of lipoproteins, as shown in Figure 2. Quantitative analysis of the TC contents of these fractions consistently showed two prominent changes in transgenic rabbits compared to control rabbits. Transgenic rabbits had significantly lower levels (30\% reduction) of large apoB-containing lipoproteins $(d<1.006 \mathrm{~g} / \mathrm{ml}, \beta$-VLDL) but concomitantly higher levels of large LDL $(d=1.02-$ $1.04 \mathrm{~g} / \mathrm{ml})(207 \%$ increase $)$ and small LDL $(d=1.04-$ $1.06 \mathrm{~g} / \mathrm{ml})(385 \%$ increase) than did control rabbits, while the levels of IDLs $(d=1.006-1.02 \mathrm{~g} / \mathrm{ml})$ were not significantly changed (Figure 2a). Since transgenic and control rabbits had basically similar levels of plasma TC, we were able to compare the distribution of TC among the various lipoprotein

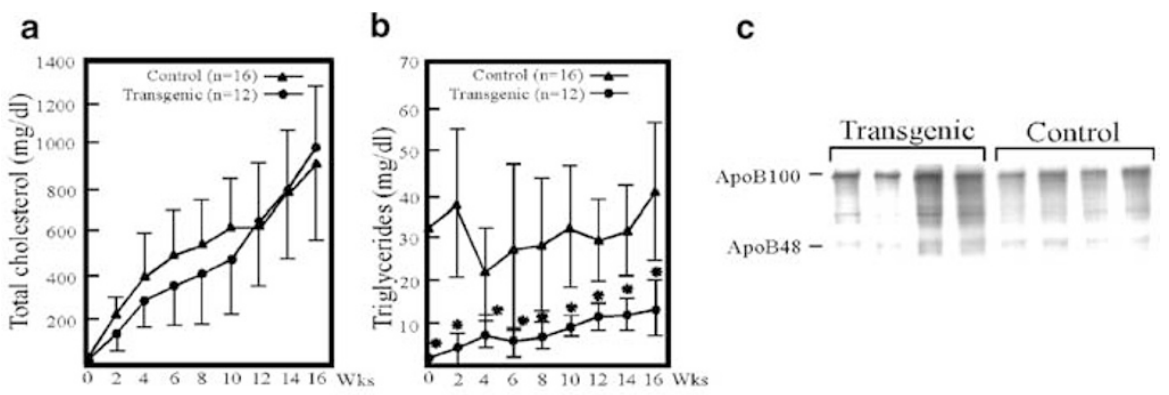

Figure 1 Plasma lipids of rabbits fed a cholesterol-enriched diet for 16 weeks. Plasma TC (a) and TG (b) were determined as described in Materials and methods. Values are expressed as mean \pm s.d. ${ }^{*} P<0.05$ vs control. Plasma apoB levels were quantitated by Western blot analysis (c). The total plasma $(0.3 \mu \mathrm{l})$ was applied to $4 \%$ SDS-PAGE gel and followed by reaction with apoB antibody that can react with both apoB-100 and -48. Immunoblots were scanned using a GS-710 calibrated imaging densitometer (Bio-Rad), and apoB-100 and -48 protein contents were expressed as the mean $\mathrm{OD} / \mathrm{mm}^{2}$. The apoB-100 value is $22.42 \pm 4.06$ in transgenic and $18.91 \pm 2.02$ in control, $P=0.172$ whereas the apoB-48 value is $12.88 \pm 2.01$ in transgenic and $11.89 \pm 0.33$ in control, $P=0.366$ ( $n=4$ for each group). 
a

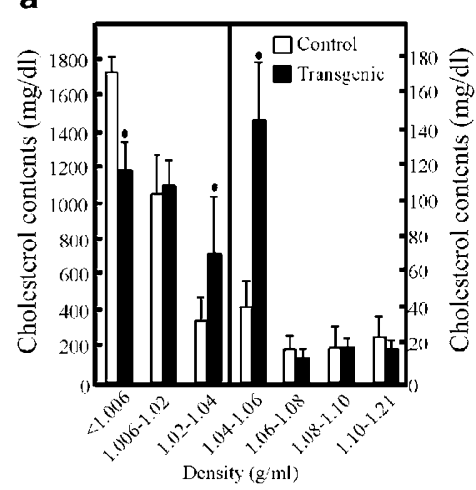

b

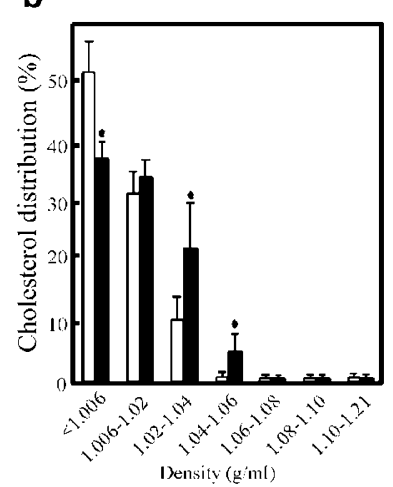

c

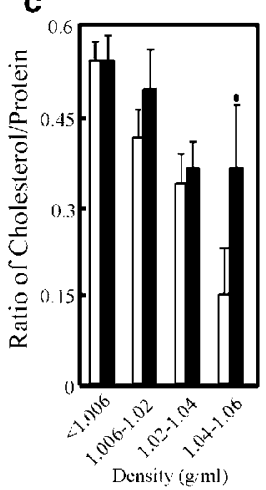

Figure 2 Quantitation of cholesterol contents in plasma lipoproteins (a). Density gradient fractions were isolated from cholesterol-fed rabbits at 16 weeks by ultracentrifugation and cholesterol contents were measured as described. The first three fractions are separated from other four fractions by a line in the middle and cholesterol contents are indicated at each side. To permit a comparison between transgenic and control rabbits, the relative distribution of cholesterol among the various fractions was plotted and expressed as percentage (b). The combined recovery of cholesterol from each animal averaged $\sim 80 \%$ of total plasma levels. Data are expressed as mean \pm s.d. ${ }^{*} P<0.01$ vs control. Furthermore, the ratio of cholesterol to proteins in four apoB-containing lipoprotein fractions was compared (c).

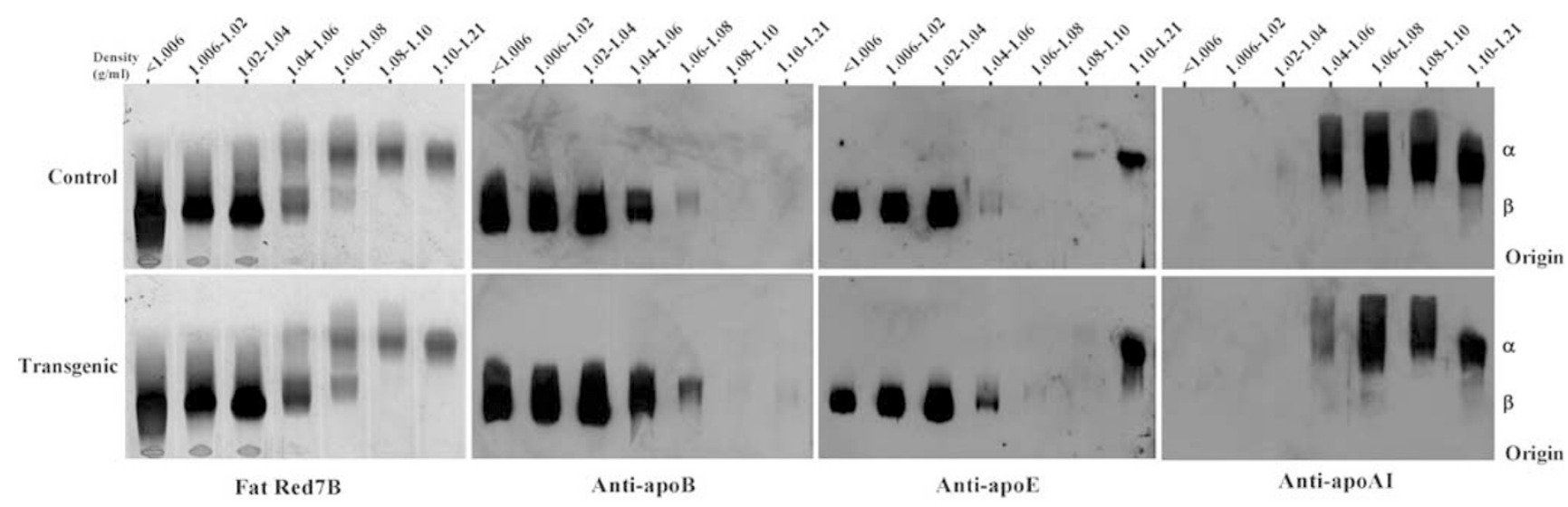

Figure 3 Plasma lipoprotein analysis. Lipoproteins were resolved by electrophoresis in a 1\% agarose gel and visualized with Fat Red 7B staining and apolipoproteins were detected by immunoblotting with specific antibodies against apoB, apoE and apoAI. Results from a representative animal in each group are shown $(n=4)$. Immunoblots were scanned using a GS-710 densitometer and apolipoprotein contents were compared. The major changes in transgenic rabbits compared to control rabbits included $150 \%$ and $120 \%$ increases of apoB and $140 \%$ and $142 \%$ increases of apoE in the $d=1.02-1.04$ and $1.04-1.06 \mathrm{~g} / \mathrm{ml}$ fractions, respectively. The apoAI content was decreased by $45 \%$ in the $d=1.04-1.06 \mathrm{~g} / \mathrm{ml}$ fraction of transgenic rabbits.

fractions. A significant reduction of $\beta$-VLDL along with increased LDLs in transgenic rabbits became noticeable when the distribution of TC among the various fractions was plotted (Figure 2b). We also measured the ratio of cholesterol to protein contents in four apoB-containing lipoprotein particles and found that small LDLs $(d=1.04-$ $1.06 \mathrm{~g} / \mathrm{ml}$ ) of transgenic rabbits contained significantly higher ratio than those of control rabbits (Figure 2c). There was, however, no significant difference in HDL fractions $(d=1.06-1.21 \mathrm{~g} / \mathrm{ml})$ between transgenic and control rabbits. The TG contents in all fractions were significantly lower in transgenic rabbits than those in control rabbits (data not shown).

The distribution of apolipoproteins among the various density fractions of lipoproteins was exam- ined by the reaction of specific Abs with Western blots of lipoproteins that had been resolved by agarose gel electrophoresis (Figure 3). The observed increase in the small LDL content in transgenic rabbits was confirmed by notable increases of apoB and apoE in these fractions. In addition, there was an increased content of smaller LDL with the density of $1.06-1.08 \mathrm{~g} / \mathrm{ml}$, where apoB was relatively increased. In the fraction with $d<1.04-1.06 \mathrm{~g} / \mathrm{ml}, \alpha-$ migrating and apoAI-containing particles (HDLc) were reduced in transgenic rabbits. These changes were also confirmed by SDS-PAGE (data not shown). Taken together, these data showed that in transgenic rabbits with equally high hypercholesterolemia to that of control rabbits, there was a significant reduction of $\beta$-VLDL, but an increase of LDL, especially small LDL particles. 


\section{Electron Microscopic Evaluation of Lipoprotein Particles}

The average diameters and distribution of the lipoproteins are shown in Figure 4a. The mean size of lipoproteins with $d<1.006$ and $d=1.04-1.06 \mathrm{~g} /$ $\mathrm{ml}$ in transgenic rabbits was larger than that in control rabbits due to the appearance of large particles in transgenic rabbits. Representative micrographs of four density fractions of apoB-containing particles are shown in Figure 4b. The large particles in the $d<1.006 \mathrm{~g} / \mathrm{ml}$ fractions were postulated to be chylomicrons ( $>75 \mathrm{~nm}$ in diameter), and the small particles $(<75 \mathrm{~nm}$ in diameter) were thought to be intestinally derived or hepatically derived remnant lipoproteins. Since the absolute number of particles in the $d<1.006 \mathrm{~g} / \mathrm{ml}$ fractions was reduced in transgenic rabbits compared with control rabbits (Figure 2), a relative increase of these large particles may reflect the fact that small-sized remnant particles were presumably removed from the circulation. Of note, in the $d=1.04-1.06 \mathrm{~g} / \mathrm{ml}$ fraction, there were two groups of lipoprotein particles: the large lipoproteins (small LDLs, $>14 \mathrm{~nm}$ ) and the small lipoproteins (so-called HDLc, $<14 \mathrm{~nm}$ ), as evaluated by their electrophoretic mobility ( $\beta$ - vs pre- $\beta$-migration) and apolipoprotein compositions (apoB/apoE vs apoAI). In transgenic rabbits, small LDLs predominated in all the fractions (Figure $4 a$ and b), suggesting that LPL may either enhance small LDL formation and/or enhance the clearance of HDLc.

a

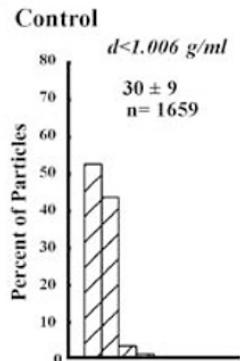

Transgenic

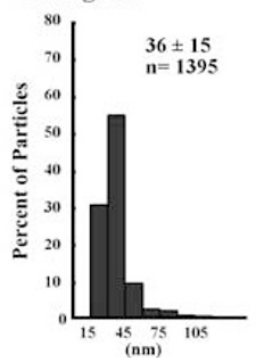

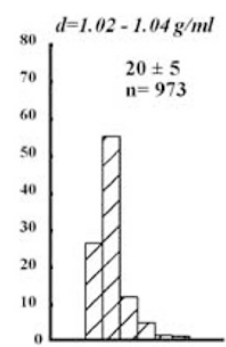
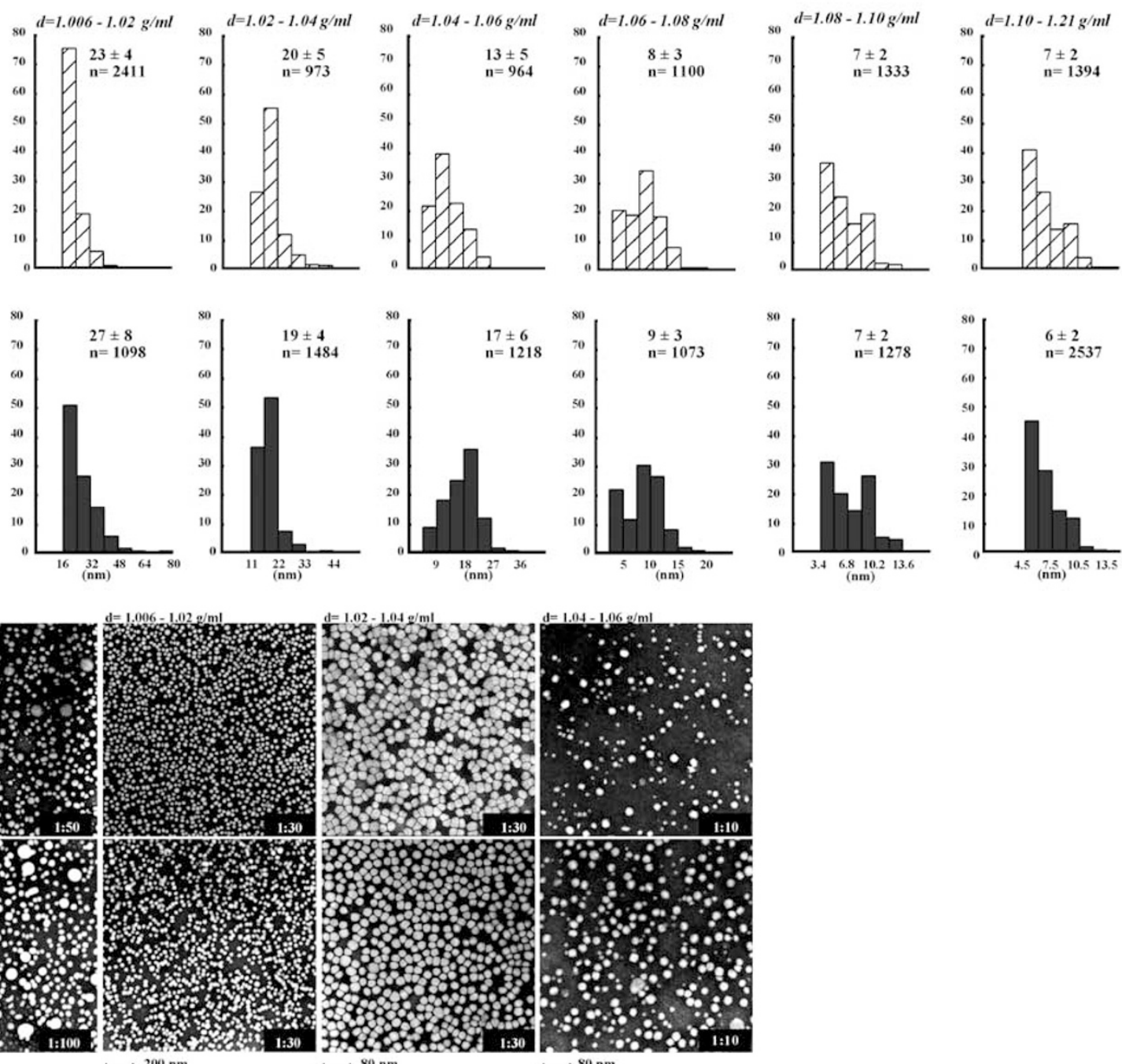

— $200 \mathrm{~nm}$

- $80 \mathrm{~nm}$

工 $80 \mathrm{~nm}$

Figure 4 Electron microscopic analysis of lipoproteins. Lipoproteins were examined by negative stain electron microscopy and measured using an image analysis system as described in Materials and methods. The distribution of the lipoproteins with various sizes is illustrated in a. The average particle diameter and the total particles counted are listed below the name of each lipoprotein class. The lipoproteins were diluted as shown at the bottom of the picture. Representative micrographs of four apoB-containing lipoproteins $(d<1.006-1.06 \mathrm{~g} / \mathrm{ml})$ are shown in $\mathbf{b}$. 


\section{Quantification of Aortic Atherosclerosis}

Since transgenic rabbits had equally high levels of plasma TC but different lipoprotein profiles (decreased $\beta$-VLDL and increased small LDL) compared with control rabbits, we examined whether the altered lipoprotein profiles were associated with atherosclerotic lesions in these animals. Analysis of sudanophilic lesions relative to the surface area revealed that transgenic rabbits had a significant increase of aortic atherosclerosis compared to control rabbits: 1.8-, 1.75- and 2.8-fold increase of atherosclerosis in the total aorta, aortic arch and thoracic aorta on average, respectively (Figure 5). Furthermore, we quantified the lesions by analyzing the sections (intimal lesion area) under a light microscope. For this purpose, we examined the aortic arch because the lesions in this area were consistently present in all cholesterol-fed rabbits. Histological examination showed that the atherosclerotic lesion areas of transgenic rabbits were twofold greater than those of control rabbits (Figure 6), although the cellular components (macrophages and smooth muscle cells) were not significantly different (data not shown). Since LPL in the arterial wall may mediate the progress of lesion development, we studied the lesions using immunohistochemical staining. In foam cell-rich lesions (fatty streak), the foam cells were mainly derived from macrophages mixed with a small number of smooth muscle cells. Oxidized LDL was invariably associated with foam cells. A small amount of LPL immunoreactive proteins were found around the extracellular matrix and colocalized with apoB (Figure 7).

\section{Analyses of Atherogenic Properties of apoB-containing Lipoproteins in vitro}

Since transgenic rabbits had more extensive lesions than control rabbits, we next sought to gain insight into the mechanism of the enhancement of athero- sclerosis in transgenic rabbits. The difference in the lipoprotein profiles between the two groups of rabbits led us to hypothesize that LDL particles (especially small LDLs) may be more atherogenic than those of remnant lipoproteins. To explore this possibility, we isolated four apoB-containing lipoprotein fractions $(d<1.006, d=1.006-1.02,1.02-$ 1.04, and 1.04-1.06 g/ml) as shown in Figure $4 \mathrm{~b}$, and compared their susceptibility to copper-induced oxidization in vitro. In control rabbits, $\beta$-VLDLs were the most sensitive to oxidization among the

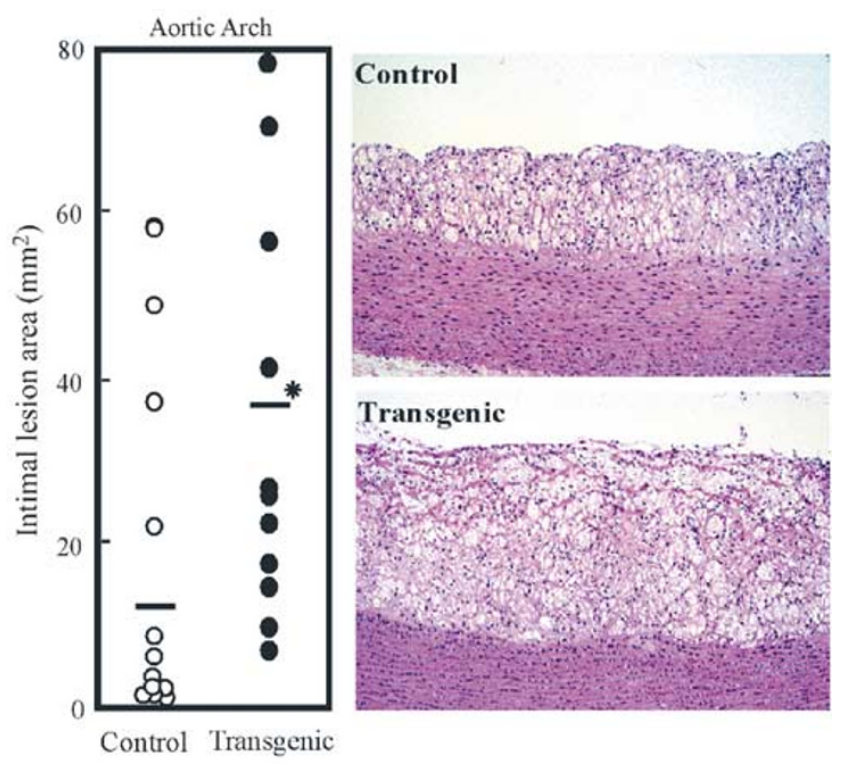

Figure 6 Microscopic analysis of lesion area of the aortic arch. Cross-sections of the aortic arch from each animal were stained with EVG and the intimal lesion area was quantitated by evaluating 10-12 serial sections at a 1-mm interval throughout the aortic arch using an image analysis system (left panel). The values are expressed as lesion size $\left(\mathrm{mm}^{2}\right)$. ${ }^{*} P<0.05$ vs control. Representative micrographs stained with hematoxylin and eosin (right panel) from each group are shown. Original magnification: $\times 40$.
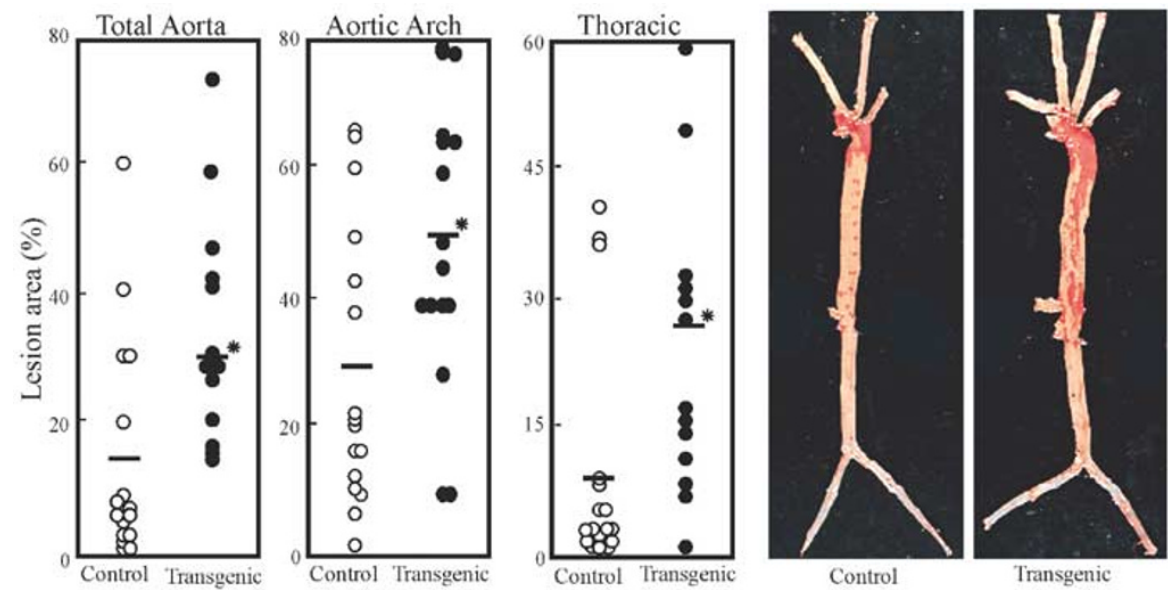

Figure 5 Quantitative analysis of aortic atherosclerosis. The whole aortic trees were stained with Sudan IV and the sudanophilic area in the entire aorta, aortic arch and thoracic aorta was measured as described in Materials and methods. ${ }^{\star} P<0.05$ vs control. Representative photographs of the pinned-out arterial trees are shown in the right panel. Sudanophilic (red) areas represent atherosclerotic lesions. 

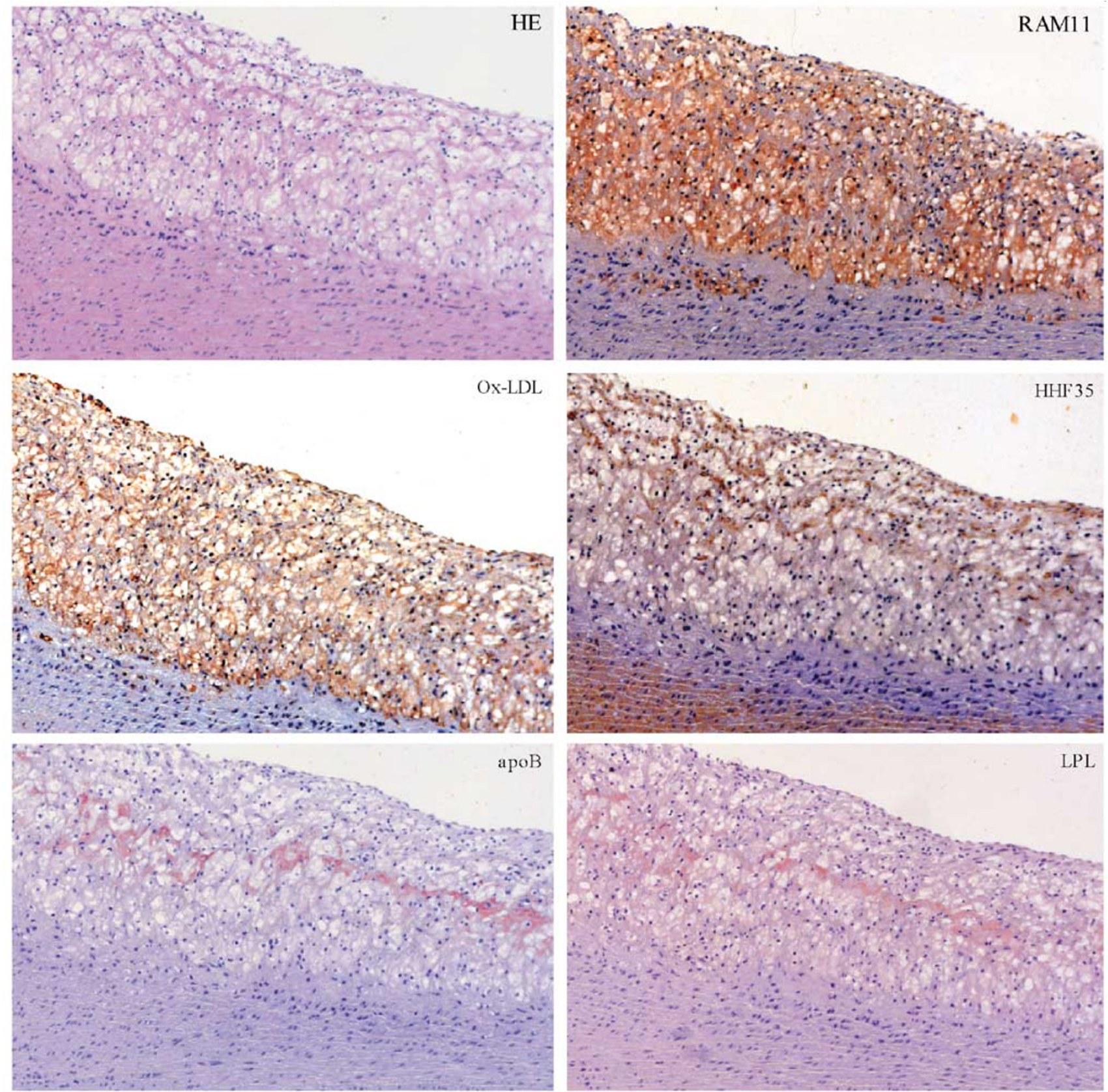

Figure 7 Analysis of the lesions by immunohistochemical staining. Serial sections of the aortic arch of a transgenic rabbit were stained with HE or Abs against macrophages, oxidized LDL, smooth muscle $\alpha$-actin, apoB and LPL. Note that LPL and apoB are colocalized. Original magnification: $\times 40$.

four fractions (Figure 8). In contrast, in transgenic rabbits, however, all LDL fractions $(d=1.006-1.06 \mathrm{~g} /$ $\mathrm{ml}$ ) were more sensitive to copper than large-sized $\beta$-VLDLs. When the comparison was made between transgenic and control rabbits, the differences between $\beta$-VLDLs and all LDL fractions were remarkable (Figure 8).

Furthermore, we compared the ability of the apoBcontaining lipoproteins to bind to biglycan, a major component of proteoglycans that are involved in atherogenic lipoprotein retention in the lesions. ${ }^{32}$ In control rabbits, the four fractions of lipoproteins showed a similar ability to bind to the biglycan matrix; however, in transgenic rabbits, there was a tendency for the smaller particles to be associated with stronger binding ability. When we compared the binding ability of each fraction isolated from transgenic rabbits with that from control rabbits, we found that the small-sized lipoproteins $(d=1.04-$ $1.06 \mathrm{~g} / \mathrm{ml}$ ) of transgenic rabbits showed apparently higher affinity to biglycan than those of control rabbits (Figure 9). 

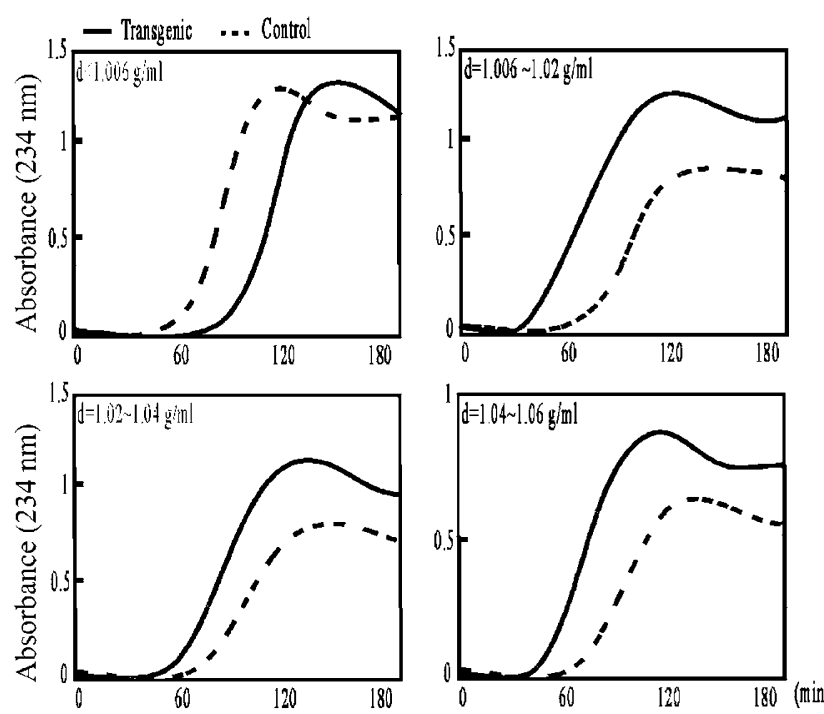

Figure 8 Kinetics of apoB-containing lipoprotein oxidation. Four apoB-containing lipoprotein fractions were isolated from cholesterol-fed rabbits as described in Materials and methods. Oxidizability was evaluated by measuring the change in absorbance at $234 \mathrm{~nm}$ as described, and lag time and diene production were calculated. Representative results $(n=3)$ are shown. In the four fractions of control and transgenic rabbits, lag time (min) was 63.7 vs $81,59.3$ vs 39.6 , 67.2 vs 56.6 , and 69.3 vs 50.6, respectively. Diene production $(\mu \mathrm{mol} / \mathrm{g})$ was 827.7 vs 842.2 , 631.5 vs 815.3, 577.9 vs 678.7 , and 427.6 vs 586.3, respectively.
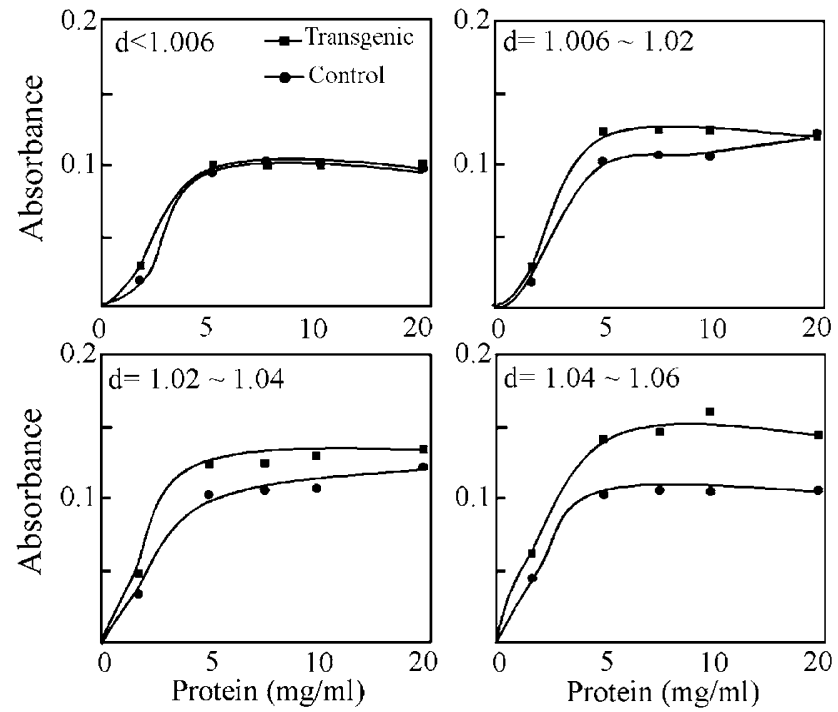

Figure 9 Assay of binding apoB-containing lipoproteins to biglycan. Binding affinity to biglycan was measured and representative results $(n=3)$ are shown. Each well was loaded with an equal amount of the indicated lipoprotein fraction and incubated for $1 \mathrm{~h}$.

Finally, we compared the ability of $\beta$-VLDLs and small LDLs to stimulate cholesteryl ester formation in macrophages. The cholesteryl ester formation induced by $\beta$-VLDLs from both transgenic and control rabbits was larger than that by their counter-

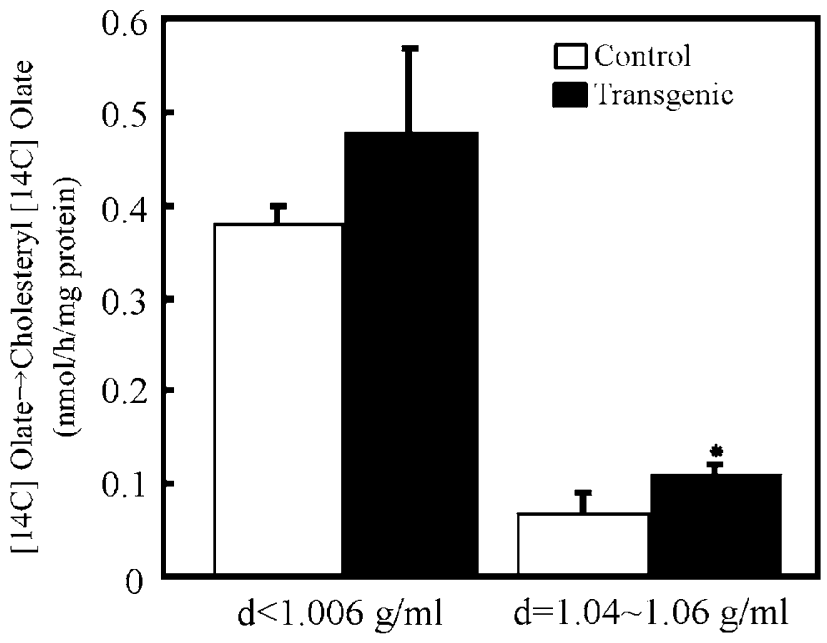

Figure 10 Cholesteryl ester formation induced by either $\beta$-VLDL or $d<1.04-1.06 \mathrm{~g} / \mathrm{ml}$ lipoproteins. Thioglycollate-elicited peritoneal macrophages were obtained from ICR mice. The cells were incubated with $12.5 \mathrm{mg} / \mathrm{ml}$ of the indicated lipoproteins for $24 \mathrm{~h}$, and cholesteryl ester formation was determined as described in Materials and methods. ${ }^{*} P<0.05$ vs control $(n=3)$.

part small LDLs, but small LDLs obtained from transgenic rabbits significantly enhanced cholesteryl ester formation compared to those from control rabbits (Figure 10).

\section{Discussion}

Previous studies demonstrated that overexpression of LPL reduces the accumulation of remnant lipoproteins in cholesterol-fed animals and thereby protects against atherosclerosis. ${ }^{6,7,16}$ These studies suggest that LPL exerts an antiatherogenic effect through the reduction of plasma remnant lipoproteins. In contrast to the previous studies in which LPL transgenic animals had dramatically lower plasma cholesterol levels than normal control animals, in the current study, we compared the lipoprotein profiles and lesions between transgenic and control rabbits that had identical TC levels. Overexpression of LPL in transgenic rabbits led to a significant reduction of large remnant particles, $\beta$ VLDL $(d<1.006 \mathrm{~g} / \mathrm{ml})$, but concomitantly led to a significant increase of LDL particles, especially those of small and dense LDLs $(d=1.06-1.08 \mathrm{~g} / \mathrm{ml})$, suggesting that LPL may enhance the process of the conversion from VLDLs to LDLs, possibly via enhanced lipolysis. The current study also allowed us to examine the hypothesis that increased LPL activity may enhance the production of smaller LDLs and thus explore intrinsic differences in the atherogenicity between animals with high levels of $\beta$-VLDLs (control rabbits) and LDLs (LPL transgenic rabbits).

We found that under conditions of equally high hypercholesterolemia, transgenic rabbits had 
significantly greater aortic lesion areas than control rabbits. These results suggest that the antiatherogenic effect of LPL reported previously in transgenic animals was basically dependent upon the plasma cholesterol level ${ }^{16}$ and that at a given level of atherogenic-cholesterol, LPL is not antiatherogenic but rather substantially proatherogenic. The mechanism(s) for the enhancement of lesion formation in LPL transgenic rabbits may be multiple, but may be related to alterations of the lipoprotein profiles. First, the lipoprotein profiles of transgenic rabbits are characterized by reduced $\beta$-VLDL and increased LDL particles, even when the TC levels are similar. Although both $\beta$-VLDL and LDLs carry cholesterol in circulation, they are not equally atherogenic once deposited in the arterial intima. ${ }^{23} \beta$-VLDLs are large in size and contain more apoB-48 in postprandial state, whereas small-sized LDLs contain more apoB100. In light of such physical and chemical differences between the two particles, one can envision that small-sized LDLs are more atherogenic because these small LDLs may enter the intima more easily and may be more susceptible to oxidation. ${ }^{34,35}$ In support of this notion, we demonstrated that small LDLs in transgenic rabbits are more sensitive to copper-induced oxidizability than $\beta$-VLDLs (Figure 8). Secondly, the increased atherosclerosis in transgenic rabbits may result from the fact that small-sized LDLs have higher affinity for proteoglycans than large remnant lipoproteins, thereby leading to the enhanced retention of these particles within the arterial intima. ${ }^{36}$ Once trapped in the intima, atherogenic lipoproteins become more susceptible to chemical modification such as oxidation, and thus may be more avidly taken up by macrophages, accelerating foam cell formation. ${ }^{37}$ To examine this possibility, we compared the binding affinity of apoB-containing particles isolated from both transgenic and control rabbits. The affinity of apoB-containing lipoproteins for biglycans was correlated with both particle size and composition. In LPL transgenic rabbits, the smaller the particles were, the higher the affinity was. Comparison between transgenic and control rabbits revealed that LDLs obtained from transgenic rabbits showed higher binding affinity to biglycan than those from control rabbits. A possible explanation for this finding is that LPL modulates apoB-containing particles through hydrolysis of TG and thus results in a shift of large LDL towards a smaller and denser population (Figure 3). Analysis of small-sized LDLs derived from transgenic rabbits revealed that these particles are substantially enriched in both apoB100 and cholesterol but devoid of TG. These chemical (ratio of proteins to lipids per particle) or conformational changes of apoB proteoglycan binding site may account for the proatherogenicity of these lipoproteins. ${ }^{36,38}$ Interestingly, LDLs from transgenic rabbits also induced a small but discernable increase of cholesteryl ester formation in macrophages even these particles are a poor ligand for LDL receptors (Figure 10). In transgenic rabbits, we detected hLPL proteins $(503 \pm 30 \mathrm{ng} / \mathrm{ml}, n=5)$ in preheparin plasma, accounting for about $\frac{1}{3}$ of hLPL present in postheparin plasma. It is currently unknown whether such preheparin LPL may be partially responsible for the increased atherosclerosis in transgenic rabbits.

Previous studies also indicated that macrophagederived LPL in the arterial wall may be potentially atherogenic. Transplantation of fetal liver cells or bone marrow of LPL-deficient mice into C57BL/6 mice or LDL receptor-KO mice reduces atherosclerosis, suggesting that LPL in the arterial wall is atherogenic. ${ }^{13,14,39}$ We also considered the possible direct impact of LPL secreted by arterial wall on the increased extent of atherosclerosis as the LPL transgene was also expressed in macrophage and aorta as detected by Northern blot analysis (data not shown). Immunohistochemical staining results showed that in transgenic rabbits, a small amount of LPL proteins was located extracellularly in the lesions. Recently, Bóren's group ${ }^{40}$ demonstrated that LPL enzymatic activity is essential for macrophagederived-LPL enhanced atherosclerosis. To elucidate directly the functional role of LPL in the arterial wall, studies using transgenic rabbits with macrophage-specific expression of LPL will be critical and we are currently performing an experiment using LPL transgenic rabbits that had macrophage-specific expression of LPL (Ichikawa et al, submitted for publication). In the current study, transgenic rabbits were fed with a diet containing higher cholesterol to adjust their plasma cholesterol levels remained similar to control rabbits. It may be likely that increased cholesterol feeding in transgenic rabbits may affect the lesion severity since this diet feeding may lead to increased cholesterol content in chylomicron remnant particles of transgenic rabbits even though these particles were not detected because of their fast clearance. We think that this diet-feeding effect on the enhanced atherosclerosis is less likely because in a separate study using LPL transgenic Watanabe heritable hyperlipidemic (WHHL) rabbits, we found these WHHL rabbits fed a chow diet had even lower levels of plasma cholesterol $(<300 \mathrm{mg} /$ dl) than control WHHL rabbits $(\sim 700 \mathrm{mg} / \mathrm{dl})$, but they had increased aortic lesions of atherosclerosis due to the strikingly increased levels of small and dense LDLs (Koike et al, unpublished data). Therefore, it is clear that small LDLs are more atherogenic than $\beta$-VLDL and may help explain the clinical finding that small LDLs are the major risk factor for coronary heart disease. ${ }^{20,41}$ Whether high LPL activity in hyperlipidemic humans is correlated with increased small LDL levels or increased risk of coronary heart disease and atherosclerosis remains to be determined.

In summary, our results, along with previous studies, suggest that increased LPL is effective in reducing remnant lipoproteins and exerts an antiatherogenic role in cholesterol-fed animals. 
Paradoxically, increased LPL activity also results in an increased production of small LDLs, which is proatherogenic when plasma cholesterol is raised. This study also indicates that small LDLs are more atherogenic than large remnant lipoproteins.

\section{Acknowledgements}

We thank Dr J Brunzell (University of Washington, Seattle) for providing anti-human LPL antibody (5D2), Dr H Itaba (Teikyo University) for providing oxLDL antibody, Drs Y Yamamoto, N Terui (Department of Chemistry, University of Tsukuba) for their help with LDL oxidization assay, Dr K Yagami (Animal Center of Tsukuba University) for animal maintenance, Dr A Miyazaki (Department of Biochemistry, Showa University) for his instruction on cholesteryl ester formation experiment, Dr J Bóren (Wallenberg Laboratory, Goteborg University) for his advice on binding assay. This work was supported in part by grants-in-aid for scientific research from the Ministry of Education, Science, and Culture of Japan and the Japan Society for the Promotion of Sciences (JSPS-RFTF 96I00202), the Mochida Memorial Foundation and Uehara Memorial Foundation, and a grant from the Center for Tsukuba Advanced Research Alliance (TARA) at the University of Tsukuba.

\section{References}

1 Eckel RH. Lipoprotein lipase. A multifunctional enzyme relevant to common metabolic disease. N Engl J Med 1989;320:1060-1068.

2 Brunzell J. Familial lipoprotein lipase deficiency and other causes of the chylomicronemia syndrome. In: Scriver C, Beaudet A, Sly W, Valle D (eds). McGrawHill: New York, 1995, pp 1913-1932.

3 Goldberg IJ. Lipoprotein lipase and lipolysis: central roles in lipoprotein metabolism and atherogenesis. J Lipid Res 1996;37:693-707.

4 Tsutsumi K, Inoue Y, Shima A, et al. The novel compound NO-1886 increases lipoprotein lipase activity with resulting elevation of high density lipoprotein cholesterol, and long-term administration inhibits atherogenesis in the coronary arteries of rats with experimental atherosclerosis. J Clin Invest 1993;92: 411-417.

5 Chiba T, Miura S, Sawamura F, et al. Antiatherogenic effects of a novel lipoprotein lipase-enhancing agent in cholesterol-fed New Zealand white rabbits. Arterioscler Thromb Vasc Biol 1997;17:2601-2608.

6 Shimada M, Ishibashi S, Inaba T, et al. Suppression of diet-induced atherosclerosis in low density lipoprotein receptor knockout mice overexpressing lipoprotein lipase. Proc Natl Acad Sci USA 1996;93: 7242-7246.

7 Yagyu H, Ishibashi S, Chen Z, et al. Overexpressed lipoprotein lipase protects against atherosclerosis in apolipoprotein E knockout mice. J Lipid Res 1999; 40:1677-1685.
8 Benlian P, De Gennes JL, Foubert L, et al. Premature atherosclerosis in patients with familial chylomicronemia caused by mutations in the lipoprotein lipase gene. N Engl J Med 1996;335:848-854.

9 Wittekoek ME, Pimstone SN, Reymer PW, et al. A common mutation in the lipoprotein lipase gene (N291S) alters the lipoprotein phenotype and risk for cardiovascular disease in patients with familial hypercholesterolemia. Circulation 1998;97:729-735.

10 O’Brien K, Gordon D, Deeb S, et al. Lipoprotein lipase is synthesized by macrophage-derived foam cells in human coronary atherosclerotic plaques. J Clin Invest 1992;89:1544-1550.

11 Araki M, Fan J, Watanabe T. Immunohistochemical localization of lipoprotein lipase and apolipoprotein $\mathrm{E}$ in human atherosclerotic lesions. Acta Histochem Cytochem 1998;31:485-492.

12 Zilversmit D. A proposal linking atherogenesis to the interaction of endothelial lipoprotein lipase with triglyceride-rich lipoproteins. Circ Res 1973;33: 633-638.

13 Babaev VR, Fazio S, Gleaves LA, et al. Macrophage lipoprotein lipase promotes foam cell formation and atherosclerosis in vivo. J Clin Invest 1999;103: 1697-1705.

14 Babaev VR, Patel MB, Semenkovich CF, et al. Macrophage lipoprotein lipase promotes foam cell formation and atherosclerosis in low density lipoprotein receptordeficient mice. J Biol Chem 2000;275:26293-26299.

15 Mann WA, Meyer N, Weber W, et al. Apolipoprotein E and lipoprotein lipase co-ordinately enhance binding and uptake of chylomicrons by human hepatocytes. Eur J Clin Invest 1995;25:880-882.

16 Fan J, Unoki H, Kojima N, et al. Overexpression of lipoprotein lipase in transgenic rabbits inhibits dietinduced hypercholesterolemia and atherosclerosis. J Biol Chem 2001;276:40071-40079.

17 Saxena U, Klein M, Vanni T, et al. Lipoprotein lipase increases low density lipoprotein retention by subendothelial cell matrix. J Clin Invest 1992;89:373-380.

18 Beisiegel U, Weber W, Bengtsson-Olivecrona G. Lipoprotein lipase enhances the binding of chylomicrons to low density lipoprotein receptor-related protein. Proc Natl Acad Sci USA 1991;88:8342-8346.

19 Rumsey SC, Obunike JC, Arad Y, et al. Lipoprotein lipase-mediated uptake and degradation of low density lipoproteins by fibroblasts and macrophages. J Clin Invest 1992;90:1504-1512.

20 Austin MA, Breslow JL, Hennekens C, et al. Lowdensity lipoprotein subclass patterns and risk of myocardial infarction. JAMA 1988;260:1917-1921.

21 Krauss RM. Dense low density lipoproteins and coronary artery disease. Am J Cardiol 1995;75: $53 \mathrm{~B}-57 \mathrm{~B}$

22 Veniant MM, Sullivan MA, Kim SK, et al. Defining the atherogenicity of large and small lipoproteins containing apolipoprotein B100. J Clin Invest 2000; 106:1501-1510.

23 Veniant MM, Withycombe S, Young SG. Lipoprotein size and atherosclerosis susceptibility in Apoe(-/-) and Ldlr(-/-) mice. Arterioscler Thromb Vasc Biol 2001;21:1567-1570.

24 Fan J, Watanabe T. Transgenic rabbits as therapeutic protein bioreactors and human disease models. Pharmacol Therapeut 2003;99:261-282.

25 Barter P. CETP and atherosclerosis. Arterioscler Thromb Vasc Biol 2000;20:2029-2031. 
26 Fan J, Ji Z-S, Huang Y, et al. Increased expression of apolioprotein $\mathrm{E}$ in transgenic rabbits results in reduced levels of very low density lipoproteins and an accumulation of low density lipoproteins in plasma. J Clin Invest 1998;101:2151-2164.

27 Fan J, Shimoyamada H, Sun H, et al. Transgenic rabbits expressing human apolipoprotein(a) develop more extensive atherosclerotic lesions in response to a cholesterol-rich diet. Arterioscler Thromb Vasc Biol 2001;21:88-94.

28 Ichikawa $\mathrm{T}$, Unoki $\mathrm{H}$, Sun $\mathrm{H}$, et al. Lipoprotein(a) promotes smooth muscle cell proliferation and dedifferentiation in atherosclerotic lesions of human apo(a) transgenic rabbits. Am J Pathol 2002;160: 227-236.

29 Itabe H, Takeshima E, Iwasaki H, et al. A monoclonal antibody against oxidized lipoprotein recognizes foam cells in atherosclerotic lesions. Complex formation of oxidized phosphatidylcholines and polypeptides. J Biol Chem 1994;269:15274-15279.

30 Koike T, Wang X, Unoki H, et al. Increased expression of lipoprotein lipase in transgenic rabbits does not lead to abnormalities in skeletal and heart muscles. Muscle Nerve 2002;26:823-827.

31 Kleinveld HA, Hak-Lemmers HL, Stalenhoef AF, et al. Improved measurement of low-density-lipoprotein susceptibility to copper-induced oxidation: application of a short procedure for isolating low-density lipoprotein. Clin Chem 1992;38:2066-2072.

32 Skalen K, Gustafsson M, Rydberg EK, et al. Subendothelial retention of atherogenic lipoproteins in early atherosclerosis. Nature 2002;417:750-754.
33 Goldstein JL, Basu SK, Brown MS. Receptor-mediated endocytosis of low-density lipoprotein in cultured cells. Methods Enzymol 1983;98:241-260.

34 Witztum JL. The oxidation hypothesis of atherosclerosis. Lancet 1994;344:793-795.

35 Steinberg D. Atherogenesis in perspective: Hypercholesterolemia and inflammation as partners in crime. Nat Med 2002;8:1211-1217.

36 Boren J, Gustafsson M, Skalen K, et al. Role of extracellular retention of low density lipoproteins in atherosclerosis. Curr Opin Lipidol 2000;11: 451-456.

37 Camejo G, Hurt-Camejo E, Wiklund O, et al. Association of apoB lipoproteins with arterial proteoglycans: pathological significance and molecular basis. Atherosclerosis 1998;139:205-222.

38 Boren J, Lookene A, Makoveichuk E, et al. Binding of low density lipoproteins to lipoprotein lipase is dependent on lipids but not on apolipoprotein B. J Biol Chem 2001;276:26916-26922.

39 Van Eck M, Zimmermann R, Groot PH, et al. Role of macrophage-derived lipoprotein lipase in lipoprotein metabolism and atherosclerosis. Arterioscler Thromb Vasc Biol 2000;20:E53-62.

40 Gustafsson M, Skalen K, Flood C, et al. Mice overexpressing enzymatic active human lipoprotein lipase (Lp) in macrophages developed more atherosclerosis than mice overexpressing inactive human LpL. Atherosclerosis 2003;4(Suppl):153.

41 Krauss RM. Is the size of low-density lipoprotein particles related to the risk of coronary heart disease? JAMA 2002;287:712-713. 